\title{
Crop Row Detection in Maize for Developing Navigation Algorithms under Changing Plant Growth Stages
}

\author{
David Reiser $^{1}$, Miguel Garrido Izard², Manuel Vázquez Arellano ${ }^{1}$, \\ Hans W. Griepentrog ${ }^{1}$ and Dimitris S. Paraforos ${ }^{1}$ \\ ${ }^{1}$ University of Hohenheim, Institute of Agricultural Engineering, \\ Garbenstr. 9, D-70599, Stuttgart, Germany \\ \{dreiser,hw.griepentrog, manuel_vazquez,d.paraforos\}@uni-ho- \\ henheim.de \\ ${ }^{2}$ Laboratorio de Propiedades Físicas (LPF)-TAGRALIA, Technical \\ University of Madrid, Madrid 28040, Spain miguel.garrido.iz- \\ ard@gmail.com
}

\begin{abstract}
To develop robust algorithms for agricultural navigation, different growth stages of the plants have to be considered. For fast validation and repeatable testing of algorithms, a dataset was recorded by a 4 wheeled robot, equipped with a frame of different sensors and was guided through maize rows. The robot position was simultaneously tracked by a total station, to get precise reference of the sensor data. The plant position and parameters were measured for comparing the sensor values. A horizontal laser scanner and corresponding total station data was recorded for 7 times over a period of 6 weeks. It was used to check the performance of a common RANSAC row algorithm. Results showed the best heading detection at a mean growth height of $0.268 \mathrm{~m}$.
\end{abstract}

Keywords: ground-truth, reference, algorithms, RANSAC, total station, LIDAR, plant position, growth status, row navigation

\section{Introduction}

Autonomous robots can have a key role in increasing sustainability and resource efficiency in food production for future world population [1]. Therefore the navigation must be planed precisely and be robust enough to deal with the changing conditions on a field. But this requires, that the machines know where the crop plants are and that they don't get destroyed by the vehicle. As most of the current crops are planted in row structures, detecting these rows is one of the basic needs for the autonomous navigation of robots in semi-structured agricultural environments. Many researches had been conducted on detecting this line structures by camera images ([2],[3]), light detection and ranging (LIDAR) laser scanner data ([4],[5],[6]), or other types of sensors. Nevertheless, precise line detection, relying on noisy sensor data, is still a challenging task for a computer algorithm due to the inherent uncertainty in the environment [6]. Humans can detect objects and shapes because of experience rather than a formal mathematical definition, like a computer algorithm does [7]. The environment has a countless number of 
variables influencing the sensors, making it hard to get the right information out of the values [8]. Aside from that, plants on the field are changing their shape rapidly, making object recognition even more challenging. First the plants are growing and, second, the conditions are changing. Therefore, there is a necessity for calibration of the algorithms before the robot is able to perform the task autonomously [6].

Also, weather and lighting conditions can already produce big changes in the results. This is especially problematic for image analysis, where alternate and discontinuous luminance usual affects the outcome [7].

To deal with these uncertainties, researchers have used simulated datasets [9], artificial plants ([9], [10]) or recorded datasets ([1],[3],[6]) to evaluate their algorithms. Since a simulation is always an approximate model of the environment, it will never cover all possibilities [8]. When recording data, the question is of how to refer to the algorithm performance. One option is to set the crop row manually [6]. The precise sensor value recording of the same plants over different growth stages, can be a good way for the later evaluation of navigation algorithms. In order to understand how algorithms behave under changing conditions in a field, it is necessary to know the pictured objects and how the sensors react on them. Therefore, it is important to know the correct plant position and parameters. In order to achieve that, the plant parameters must be mapped and referenced in every new test.

The aim of this paper is to show how algorithm analyzing could be improved using precise referenced sensor data, especially when the same plants can be investigated with the same sensors over different growth stages. For that purpose, the data set of a horizontal LIDAR is used. With the help of a highly accurate total station, all sensor data sets can be converted into the same reference frame, in order to obtain comparable results. This approach is tested by the performance evaluation of a common random sample consensus (RANSAC) line fitting algorithm [11]. The RANSAC algorithm has the advantage of being fast and robust against outliers, resulting in advanced performance when dealing with noisy sensor data. Therefore the performance at different growth stages can be precisely evaluated, by using the same reference.

\section{Materials and Methods}

\section{$2.1 \quad$ Hardware and Sensors}

A small 4-wheel autonomous robot with differential steering was used to move the sensors with manual control through the crop rows (see Fig. 1). The size of the robot platform was $500 \times 600 \times 1100 \mathrm{~mm}$. The weight of the robot is $50 \mathrm{~kg}$ and it is equipped with four motors with a total power of $200 \mathrm{~W}$. Maximum driving speed is $0.8 \mathrm{~m} / \mathrm{s}$ and the maximum static motor torque is $4 \times 2,9 \mathrm{Nm}$. The robot system is equipped with wheel encoders, a VN-100 Inertial Measurement Unit (IMU) (VectorNav, Dallas, USA) and a LMS111 2D-LIDAR laser scanner (SICK, Waldkirch, Germany). The laser scanner was mounted horizontally at the front of the robot at a height of $0.2 \mathrm{~m}$ above the ground level. All other mounted sensors had not been used in this paper.

For evaluating the precise position of the robot, the SPS930 Universal Total Station (Trimble, Sunnyvale, USA) was utilized. The total station was tracking a Trimble 
MT900 Machine Target Prism, which was mounted on top of the robot at a height of $1.07 \mathrm{~m}$ in order to guarantee always line of sight to the total station (see Fig. 1).

The robot is controlled by an embedded computer, equipped with i3-Quadcore processor with $3.3 \mathrm{GHz}, 4 \mathrm{~GB}$ RAM and SSD Hard drive. For energy supply, two 12V/48Ah batteries are providing an operating time of around $4-5 \mathrm{~h}$, depending on the load torque, task and additional weight of equipment placed on the robot platform. The total station data was sent to a Yuma 2 Rugged Tablet Computer (Trimble, Sunnyvale, USA); this tablet is equipped with an Intel Atom CPU N2600 dual-core processor with $1.6 \mathrm{GHz}, 4$ GB RAM, SSD Hard drive, and a self-sufficient battery. Connectivity to the SPS930 total station is provided by an internal $100 \mathrm{~mW}$ radio antenna at the $2.4 \mathrm{GHz}$ (IEEE 802.11) range. The Yuma 2 Rugged Tablet Computer was connected to the robot computer via serial RS232 interface for continuous data exchange.

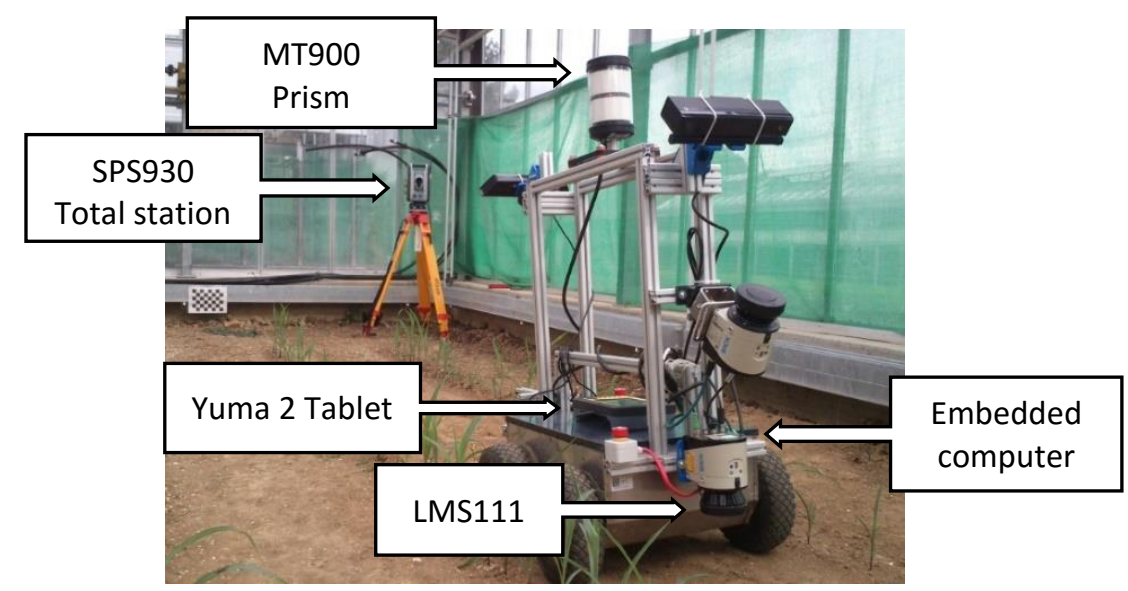

Fig. 1. Robot platform, equipped with the sensors and the reference prism

\subsection{Software}

The robot computer runs by Ubuntu 14.04 and use the Robot Operating System (ROSIndigo) middleware for the data recording. The software components had been programmed in a combination of $\mathrm{C}++$ and Python programming languages.

The Trimble Yuma 2 Tablet was running under Windows 7 Professional and executed the Trimble SCS900 Site Controller Software Version 3.4.0. The Trimble software includes an easy-to-use graphical interface for fast calibration and point measurement. It also has the option to export the actual prism position via serial RS232 interface. The tablet was placed on the robot and the serial output was directly used by the ROS system to refer the robot position to the total station coordinate frame. The prism position data was time stamped, according to the computer system time, together with the sensor data. The data flow diagram can be seen in Fig. 2. 


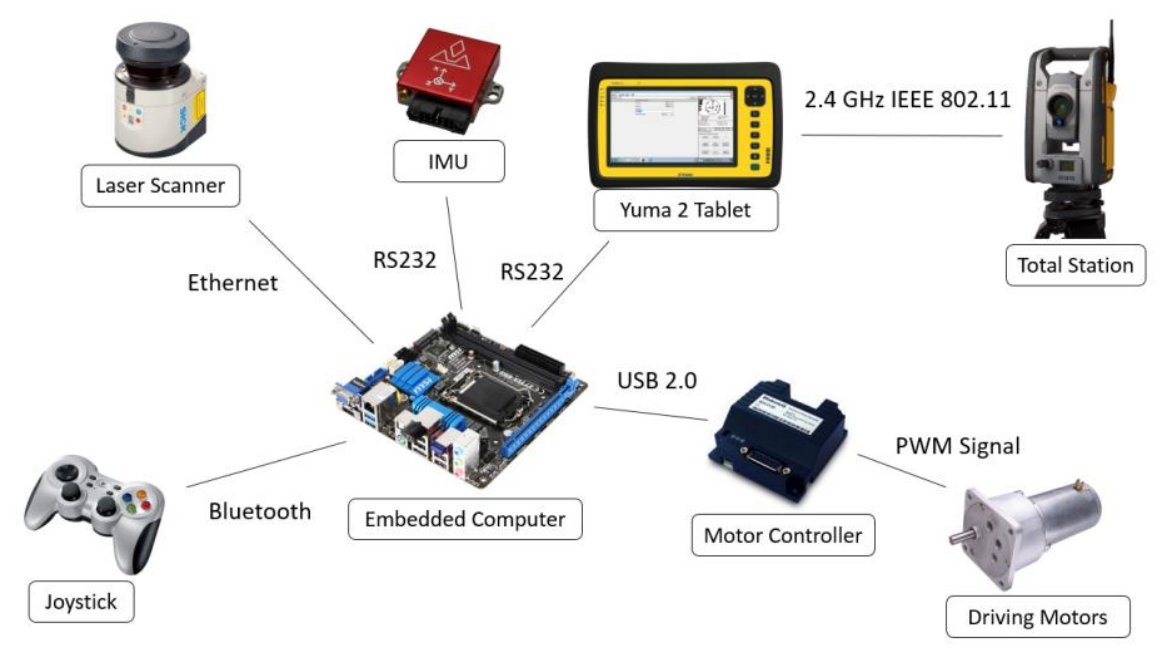

Fig. 2. Data flow diagram of the robot sensor setup

\subsection{Referencing \& Data Acquisition}

To create a relative coordinate frame in the greenhouse, five fixed attachments for the MT1000 Prism had been placed on predefined positions. The accurate position of these five points could be located by just screwing the prism on the attachments for every new field test. The absolute point distances were stored during the first setup. Before every subsequent data acquisition, these points were measured once again by the total station in order to recalibrate the system to the first total station position with the original Cartesian coordinate frame. After every test, the inaccuracy in the static measurement was evaluated by reassessing each of these fixed points. The shift between the first reference points and the actual measurement was evaluated by the Trimble SPS software and was in all tests below $4 \mathrm{~mm}$ for all three dimensions. The total station was always placed almost at the same position inside the greenhouse, which lies around the zero point of the coordinate frame.

After plant emergence, the stem position was measured with the aid of pendulum hanging from a tripod; the MT1000 Prism was attached at the center of the tripod. It was assumed that the center of each plant stem's position will not change during the period of growth. Consequently, each of these points was used as the reference position of each individual plant.

Due to the robot rigid body frame that was carrying the sensors, a static transformation between the prism and the sensor positions was performed. The three-dimensional orientation of the robot in space was evaluated by the IMU, which was placed at the center of the robot and on the same axis as the prism. As the orientation of the prism could not be evaluated by the total station, the position of the prism was fused with the IMU data to transform the laser scans to the greenhouse frame. 
This procedure allowed to directly transform the recorded sensor data into the same coordinate frame, and even to assign them to single plant positions. In Fig. 3a the test environment with the moving robot is presented and in Fig. $3 \mathrm{~b}$ the corresponding sensor value visualization of all attached sensors in the ROS environment is illustrated. The blue points correspond to the horizontal laser scanner data.

(a)

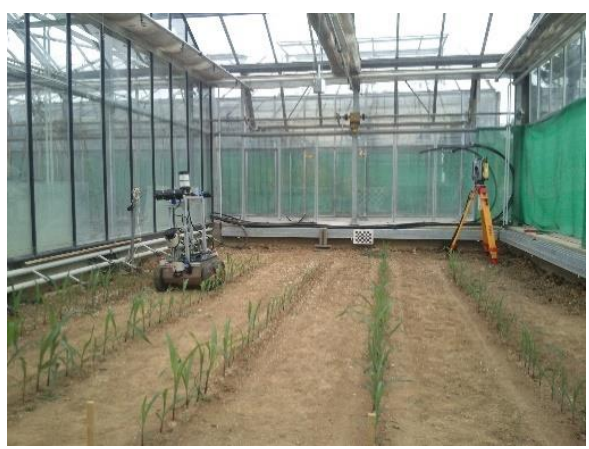

(b)

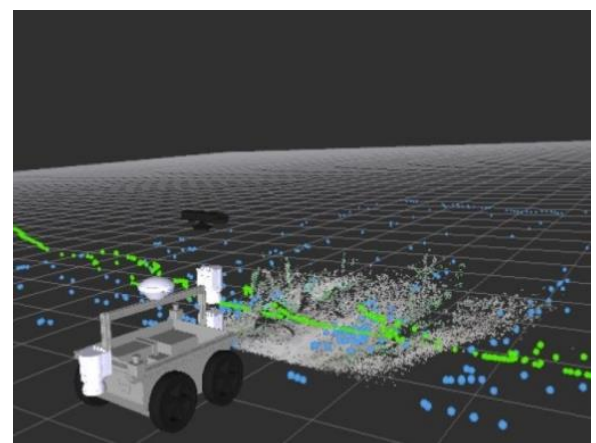

Fig. 3. (a) the greenhouse environment and (b) a visualisation of the transformed sensor data in the ROS visualisation environment

The sensor files were separated at a size of $4 \mathrm{~GB}$. The timestamp was according to the robot embedded computer system time, with a resolution of one millisecond. The LIDAR data was collected with an average of $25 \mathrm{~Hz}$ and a resolution of 0.5 degree. The total station updated the data with $15 \mathrm{~Hz}$. The IMU data was transmitted with $40 \mathrm{~Hz}$. Linear interpolation was used to fuse robot position and the sensor data before transforming it to the global coordinates of the greenhouse frame.

\subsection{RANSAC Algorithm}

The RANSAC is a commonly used algorithm for evaluating plane parameters in noisy point cloud data [12], [13]. But also for row or line estimation in image analysis [1], and 2D-LIDAR data [14], [15]. To evaluate the general algorithm behavior, the RANSAC was chosen due to its previously mentioned robustness against outliers. The implemented RANSAC algorithm is part of the Point Cloud Library [16] and was integrated in the ROS environment, for direct analysis of the published scans. To get always precise reference of the extracted lines, the LIDAR data was first transformed to the robot body frame and then to the overall greenhouse frame.

As the distance between maize crop rows is $0.75 \mathrm{~m}$, this parameter was used to filter roughly the row area with the known robot position with a rectangle. The resulting point cloud was separated to have for each crop row an individual point cloud. This was done by using the known robot position and robot direction. The RANSAC was then applied to each distinct point cloud. The maximum iteration limit was set to the input point 
number and the maximal distance range for the line to $0.5 \mathrm{~m}$. These parameters were fixed for all performed line fittings.

\section{Experiments}

Five rows of maize were planted with a length of $5.2 \mathrm{~m}$ each. The row spacing was defined according to common agricultural practice to $0.75 \mathrm{~m}$, with 41 plants per row. The maize was planted in a greenhouse to be independent of external weather conditions. The measured positions of the plants, total station and reference points can be found in Fig. 4. After every data acquisition, the height, stem width and leaf numbers of each single plant had been measured. This was done manually with a measuring tape and a sliding caliper.

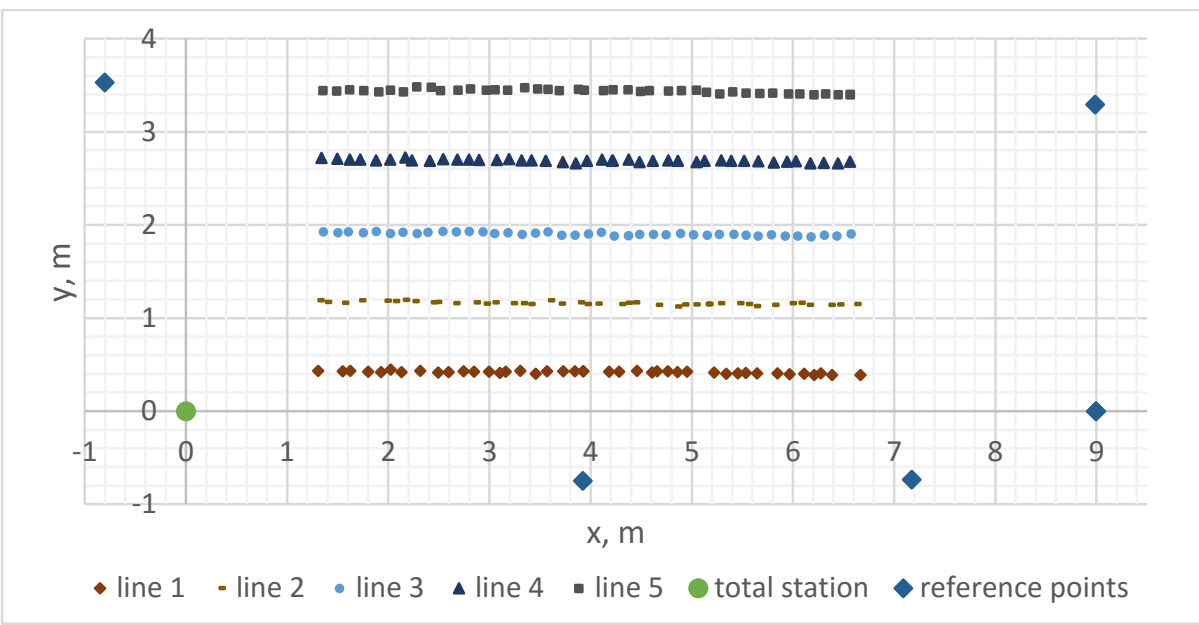

Fig. 4. Manually measured plant positions, reference points and total station position

The ideal line parameters were evaluated by the plant germination positions, measured with the total station. Because this line has no outliers, the least square algorithm can result in the most accurate line fitting. As reference, a 2D line in the XY-plane was estimated using the equation $f(x)=a x+b$. The residual $r$ of every data point $P_{i}\left(x_{i}, y_{i}\right)$ can be described with:

$$
r_{i}=f\left(x_{i}\right)-a * x_{i}-b
$$

Using the least square estimation the best line fit can be estimated as:

$$
\min _{a b} \sum_{i=1}^{n} r_{i}{ }^{2}
$$


This was adapted to the emerging points of the plants, resulting in the line parameters presented in Table 1.

Table 1: Listing of the line parameters

\begin{tabular}{ccc}
\hline line number & line equation in $[\mathrm{m}]$ & $\begin{array}{c}\text { intersection point with last row } \\
\text { in }[\mathrm{m}]\end{array}$ \\
\hline 1 & $f(x)=-0.0062 x+0.4441$ & - \\
2 & $f(x)=-0.0079 x+1.1913$ & $P(439.53,-2.28)$ \\
3 & $f(x)=-0.0085 x+1.9414$ & $P(1250.17,8.69)$ \\
4 & $f(x)=-0.0068 x+2.7186$ & $P(-457.18,5.83)$ \\
5 & $f(x)=-0.0101 x+3.4811$ & $P(231.06,1.15)$ \\
\hline
\end{tabular}

As it can be seen in the intersection points, shown in table 1, the row with the smallest angle difference between the lines is the path between crop row 2 and 3; here, the intersection point had the longest distance to the row center. The best performance was expected from the most parallel lines for evaluating the row detection algorithm. So crop row line 2 and 3 were selected. The sensor data recording took place from 23.04.15 until 1.06.15 in Stuttgart Hohenheim. In total 7 tests were performed. In every test, the robot was driven by a remote joystick with a constant speed through the crop rows. The average speed was around $0.05 \mathrm{~m} / \mathrm{s}$ in order to acquire a high data density. Both rows were recorded twice, once for each travel direction. For all 7 test days, each travel direction was evaluated, resulting in a total of 14 recorded and analyzed datasets. The laser scanner data was used, when the robot reference prism was in an area between 2 to $5 \mathrm{~m}$ in the $\mathrm{x}$ direction. For filtering purposes, the scans were first transformed to Cartesian coordinates and then to the greenhouse coordinate frame. The reflected points of the robot vehicle were removed and the point cloud was separated as described above. By doing this, the RANSAC could be performed for each crop line separately. Each of the line fittings was addressed directly to one single point cloud set without using any prior knowledge about the last dataset or the robot position. Scans with less than three points in the line area were ignored. In total 10277 different laser scans were evaluated. In Table 2 the number of analyzed scans per line are presented.

Table 2: Numbers of analyzed scans per line

\begin{tabular}{ccccc}
\hline $\begin{array}{c}\text { Test Num- } \\
\text { ber: }\end{array}$ & Date & $\begin{array}{c}\text { Days after } \\
\text { seeding }\end{array}$ & $\begin{array}{c}\text { Analyzed scans } \\
\text { line 3 }\end{array}$ & $\begin{array}{c}\text { Analyzed scan } \\
\text { line 2 }\end{array}$ \\
\hline 1 & 23.04 .2015 & 28 & 67 & 688 \\
2 & 27.04 .2015 & 32 & 601 & 990 \\
3 & 30.04 .2015 & 35 & 910 & 1041 \\
4 & 05.05 .2015 & 40 & 897 & 941 \\
5 & 13.05 .2015 & 48 & 754 & 763 \\
6 & 18.05 .2015 & 53 & 653 & 652 \\
7 & 01.06 .2015 & 67 & 660 & 660 \\
\hline
\end{tabular}


The difference between ideal line and the algorithm solved line, was evaluated with the help of the Root Mean Square Error (RMSE), defined by the following equation:

$$
\boldsymbol{R M S E}=\sqrt{\frac{\sum_{i=1}^{n}\left(\delta-\beta_{i}\right)^{2}}{n}}
$$

With $\delta$ as ideal line parameters and $\beta_{i}$ as the resolved algorithm parameters at the scan $i$.

\section{$4 \quad$ Results and Discussion}

As the greenhouse soil was not homogeneous, there was a huge diversity in growth status. For tracking the crop development, the highest point of each plant was measured and the mean value was evaluated for each crop row. The variability is expressed by the standard deviation of all 41 plants heights per crop row. The results are shown in Fig. 5. The average height of the plants at line 3 had been lower than at line 2. 48 days after seeding, most of the plants reached the level of $0.2 \mathrm{~m}$ height. At all tests afterwards the number of analyzed scans had been almost the same for both sides (see Table 2). As in the first two tests the average plant height of line 3 was below the height of the laser scanner, the RANSAC algorithm for line 3 detected points, just when the vehicle was turned downwards, because of uneven ground. The absolute mean value for the height of the line 3 was $0.47 \mathrm{~m}$ while for line 2 the mean value was $0.65 \mathrm{~m}$. The tallest plant reached $0.82 \mathrm{~m}$ at line 3 and $0.86 \mathrm{~m}$ at line 2 . For later growth stages the standard deviation was increased. Along with the height, the numbers of leaves, covering the row, were also increased. This caused limited sight of view for the LIDAR.

(a)

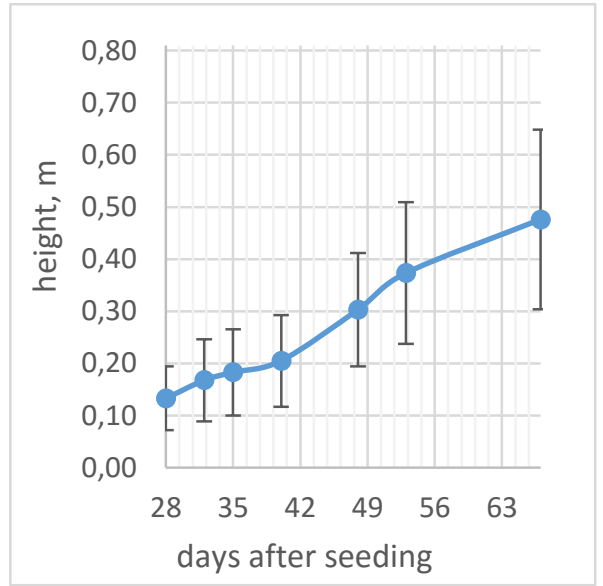

(b)

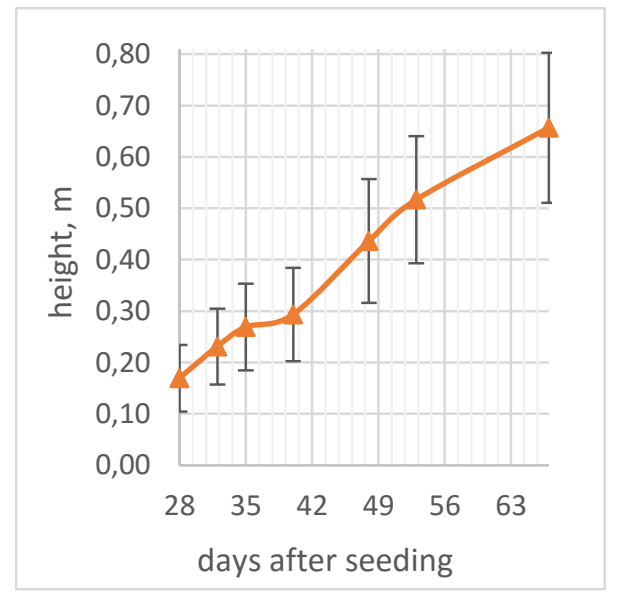

Fig. 5. Mean growth status of the plants for (a) line 3 and (b) line 2 
For evaluating the change between crop row and RANSAC output, the RMSE for every data set was estimated. The results for the row position (Fig. 6a) showed a higher error in the first tests for all mean values below $0.2 \mathrm{~m}$. For all other heights, the RMSE value fluctuated at a value around $0.1 \mathrm{~m}$. The best matches were after 35 and 67 days at line 2 with an RMSE value of $0.07 \mathrm{~m}$ and $0.06 \mathrm{~m}$, respectively.

Also the heading error (Fig 6b) had a higher value at the first tests with low mean plant height. For both lines a local minima could be detected after a 35 days (see Fig. 6b). As it could be seen in Table 2, this was the first test with almost equal number of detected lines out of the scans. With the growth of the plants, the precision decreased back again. Only the last measurement of line 3 did performed better than the first minima of the same line. A reason for this could be the inhomogeneous growth of the crop plants. In total the RANSAC performed better at line 2 than in line 3 . Reasons for that could be the more homogenous growth of the plants, which is expressed by the standard deviation of the two lines (see Fig. 5). Especially line 2 had almost constant RMSE values between 35 and 53 days after seeding.

(a)

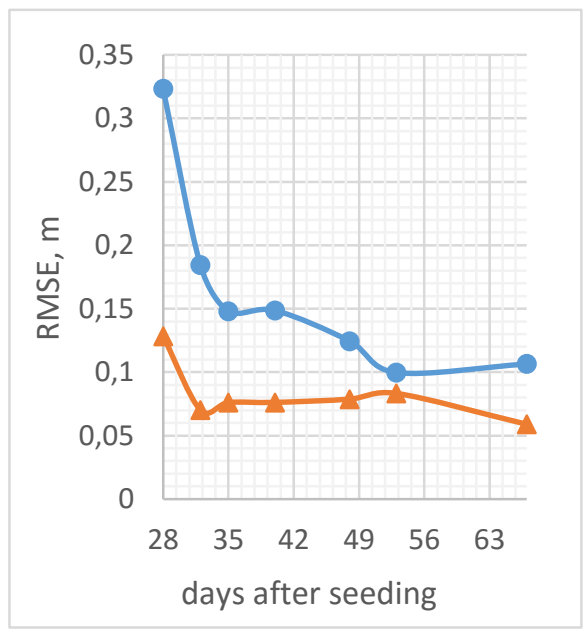

(b)

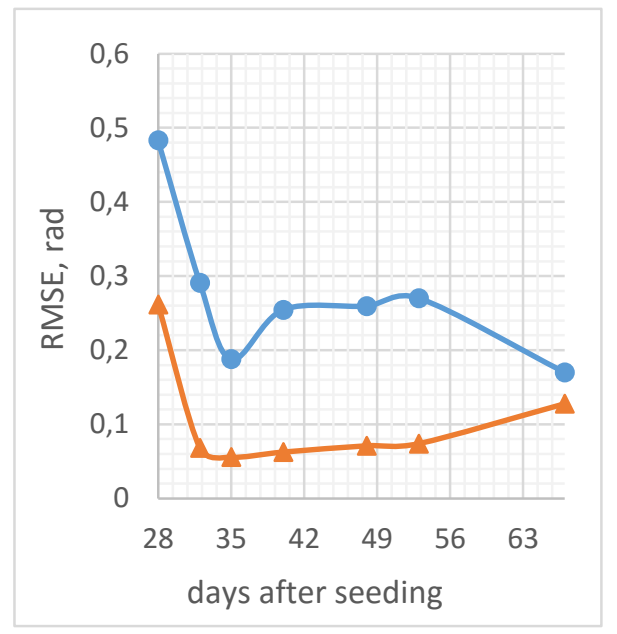

Fig. 6. RMSE of (a) position and (b) heading of row line $2(\boldsymbol{\Delta})$ and line $3(\bullet)$.

To better understand the evaluated error of the real line parameters, the direct RANSAC output is shown in Fig. 7. For evaluating the values, two tests after 35 days and two tests after 67 days are visualized. For both test days, the robot moved through the row in each direction once. 200 measurements were evaluated and compared in the following graph (see Fig. 7). The RANSAC heading output after 35 days is shown in Fig 7a and the output after 67 days can be seen in Fig $7 \mathrm{~b}$. The inclination parameter of line 2 is -0.0079 , which under ideal conditions should be the same like the computed RANSAC parameter. The nearest heading to this theoretical value can be seen at 35 days after seeding (see Fig 6b). After 67 days, the computed values increased and produced out of these a bigger shift to the reference value. At the end of the row a higher 
shift can be noticed. A reason for this could be, the lower number of points, detectable at the end of a row, to balance the outliers. After 67 days this performance was worse compared to the values after 35 days as illustrated in Fig. 7b. First this could be reasoned, by the high amount of leaves hanging into the laser scan area. These leaves blocked the detection of the stem positions that were necessary to evaluate the line orientation.

For every direction the robot moved, a static shift of the heading was observed (see Fig. 7). This can be explained by some reflections of leaves, which caused a shift of the detected line to the middle of the row. This effect was stronger after 67 days and caused a narrow detection of the plant stems. The minimal reached RMSE was 0.05 rad for the line detection with the RANSAC after 37 days. After 67 days this value increased.

In worst cases the noise could be much higher than 5 degrees $(0.1 \mathrm{rad})$ compared to the real value. This can cause problems on line following, especially when there is not enough space between the vehicle and the rows. The failure rate could be seen in many cases of the evaluated data. A part of the analyzed error could also be resulted by the inaccuracy of the LIDAR measurements.

For getting a RANSAC algorithm robust for navigation, this heading uncertainty must be compensated. Higher algorithm robustness could be accomplished using a Kalman filter. When the growth status is known, the heading error could also be decreased by a static offset, which must be evaluated before starting the line following. Filtering for outliers or mean filter methods could also bring better results.

(a)

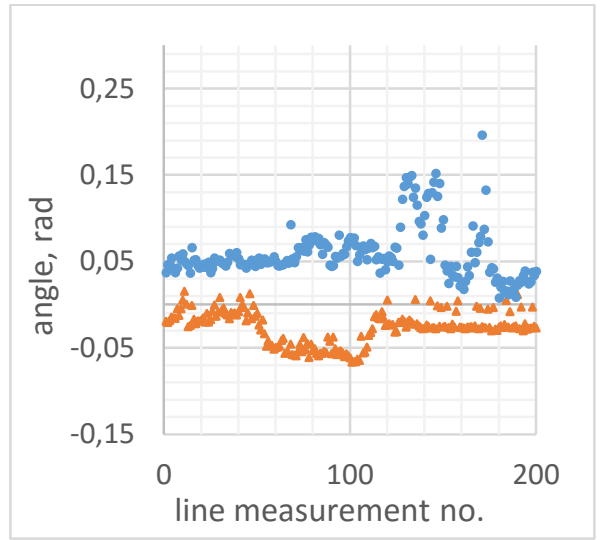

(b)

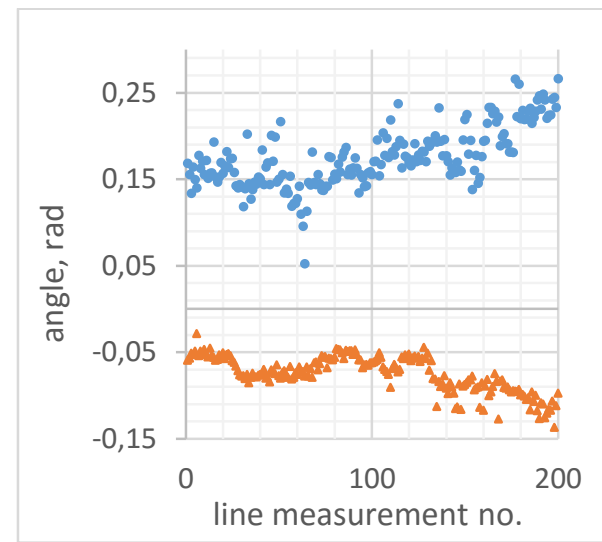

Fig. 7. Heading values of the RANSAC for (a) line 2, 35 days after seeding and (b) 67 days after seeding. Robot movement in positive $\mathrm{x}$ direction (orange triangles) and in negative $\mathrm{x}$ direction (blue dots).

The results of the RANSAC showed a high variability in the dataset, with different outcomes of the algorithm. So it could be assumed, that the variability in the dataset brings additional options for testing the robustness of line following algorithms for different growth states. The experimental setup allowed to detect a heading offset, which 
is dependent on the growth status of the crop plants. It could be shown that this offset is dependent on the sensor position and the movement of the robot. Also the best height for the line detection with the given laser position could be evaluated. This was at a mean height of $0.265 \mathrm{~m}$ of the plants.

\section{Conclusions}

The results of the collected data set showed high precision and good referenced sensor data for all measured growth stages. The application of a RANSAC algorithm for line detection to the horizontal laser data showed high diversity in heading and positioning. The smallest heading error was detected 35 days after seeding and at an average plant height of $0.268 \mathrm{~m}$. After that, the error increased and brought a higher RMSE value to the detection. Also a drift dependent on the travel direction of the robot was observed, which was caused by leaves inside the row. This effect increased with the growth of the plants. In many cases of the given data set, the deviation of the line heading was higher than 5 degrees. This would cause problems for precise row navigation. The position error was for most cases acceptable. For line following applications in maize with a RANSAC algorithm, robust filtering of the laser data and algorithm results should be considered. In total the approach was helpful in order to evaluate some basic problems of outdoor line detection with LIDARs and a RANSAC algorithm. Aside of that, the accurate reference of the heading difference could be evaluated.

\section{Acknowledgments}

The project is conducted at the Max-Eyth Endowed Chair (Instrumentation \& Test Engineering) at Hohenheim University (Stuttgart, Germany), which is partly grant funded by the DLG e.V.

\section{References}

1. English, A., Ross, P., Ball, D., Corke, P.: Vision Based Guidance for Robot Navigation in Agriculture. In: IEEE International Conference on Robotics and Automation (ICRA). pp. 1693-1698 (2014).

2. Marchant, J., Brivot, R.: Real-Time Tracking of Plant Rows Using a Hough Transform. Real-Time Imaging. 1, 363-371 (1995).

3. Jiang, G., Zhao, C., Si, Y.: A machine vision based crop rows detection for agricultural robots. In: Proceedings of the 2010 International Conference on Wavelet Analysis and Pattern Recognition. pp. 11-14 (2010).

4. Hansen, S., Bayramoglu, E., Andersen, J.C., Ravn, O., Andersen, N.A., Poulsen, N.K.: Derivative free Kalman filtering used for orchard navigation. In: 13th international Conference on Information Fusion (2010).

5. Barawid, O.C., Mizushima, A., Ishii, K., Noguchi, N.: Development of an Autonomous Navigation System using a Two-dimensional Laser Scanner in an Orchard Application. Biosyst. Eng. 96, 139-149 (2007). 
6. Hiremath, S.A., van der Heijden, G.W.A.M., van Evert, F.K., Stein, A., ter Braak, C.J.F.: Laser range finder model for autonomous navigation of a robot in a maize field using a particle filter. Comput. Electron. Agric. 100, 41-50 (2014).

7. Papari, G., Petkov, N.: Edge and line oriented contour detection: State of the art. Image Vis. Comput. 29, 79-103 (2011).

8. Russell, S.J., Norvig, P.: Artificial Intelligence: A modern approach. Ptrentice-Hall, Englewood Cliffs, NJ (1995).

9. Weiss, U., Biber, P.: Plant detection and mapping for agricultural robots using a 3D LIDAR sensor. Rob. Auton. Syst. 59, 265-273 (2011).

10. Bochtis, D., Griepentrog, H.W., Vougioukas, S., Busato, P., Berruto, R., Zhou, K.: Route planning for orchard operations. Comput. Electron. Agric. 113, 51-60 (2015).

11. Fischler, M. a, Bolles, R.C.: Random Sample Consensus: A Paradigm for Model Fitting with Applications to Image Analysis and Automated Cartography. Commun. ACM. 24, 381-395 (1981).

12. Choi, S., Park, J., Byun, J., Yu, W.: Robust Ground Plane Detection from 3D Point Clouds. In: 14th International Conference on Control, Automation and Systems (ICCAS 2014). pp. 1076-1081 (2014).

13. Weiss, U., Biber, P., Laible, S., Bohlmann, K., Zell, A.: Plant species classification using a 3D LIDAR sensor and machine learning. Proc. - 9th Int. Conf. Mach. Learn. Appl. ICMLA 2010. 339-345 (2010).

14. Zhang, J., Maeta, S., Bergerman, M., Singh, S.: Mapping Orchards for Autonomous Navigation. In: ASABE Annual International Meeting. pp. 1-9 (2014).

15. Marden, S., Whitty, M.: GPS-free Localisation and Navigation of an Unmanned Ground Vehicle for Yield Forecasting in a Vineyard. In: Proceedings of the 13th International Conference IAS-13 (2014).

16. Rusu, R.B., Cousins, S.: 3D is here: point cloud library. IEEE Int. Conf. Robot. Autom. 1-4 (2011). 